\title{
Prognostic information for known genetic carriers of RB1 pathogenic variants (germline and mosaic)
}

M. Ashwin Reddy ${ }^{1,2}$ FRCOphth MD (Res) , Mussa Butt ${ }^{1}$ MBBS, Anne-Marie Hinds ${ }^{1}$ FRCOphth, Catriona Duncan ${ }^{1,3}$ MRCPCH, Elizabeth A Price ${ }^{4}$ PhD, Mandeep S. Sagoo ${ }^{1,2}$ FRCS (Edin) PhD and Zerrin Onadim ${ }^{4}$ PhD.

${ }^{1}$ Retinoblastoma Service, Royal London Hospital, London, UK

2 NIHR Biomedical Research Centre for Ophthalmology at Moorfields Eye Hospital and University College London, Institute of Ophthalmology, London, UK

${ }^{3}$ Paediatric Oncology Department, Great Ormond Street Hospital, London, UK

${ }^{4}$ Retinoblastoma Genetic Screening Unit, Royal London Hospital, London, UK

\section{Corresponding Author}

M. Ashwin Reddy, Dept of Ophthalmology, Royal London Hospital, Whitechapel

Road, London, UK E1 1FR ashwin.reddy4@nhs.net 
66

\section{Abstract}

Objective: To compare the number of tumors per eye for mosaic carriers of $R B 1$ pathogenic variants with full germline variants and the conversion from unilateral to bilateral disease.

Design: Retrospective cohort study comparing patients with retinoblastoma and different genetic subtypes (HP: high penetrant, LP: low penetrant \& mosaicism).

Subjects: Data were analysed between 1992 and 2018 at the Retinoblastoma Unit, Royal London Hospital, London UK. All familial patients had a parent with a known pathogenic variant even if the parent did not manifest the disease.

Main outcome measures: Number of tumors per eye in children who developed retinoblastoma in that eye. Other outcomes included total number of tumors per patient, age at diagnosis, laterality at presentation and later, sex and stage according to International Intraocular Retinoblastoma Classification

Results: 111 patients were included: 64 full germline, familial patients (53 HP and 11 LP) \& 47 were mosaic patients. $12(23 \%)$ of HP patients were unilateral and 8 of $12(67 \%)$ developed tumors in their previously unaffected eye. $34(72 \%)$ of mosaic patients were unilateral and only $2(6 \%)$ developed tumors in their unaffected eye. Age at diagnosis was higher in mosaic patients (median 22 months) than HP patients (median 7$)(p<0.00002)$. Number of tumors per eye was fewer in patients with mosaic alleles (median 1.0 range 1-6) compared to patients with HP alleles (median 3.0 range $1-8)(p<0.0003)$. All three children (4 eyes) with mosaicism and more than 2 tumors per eye had high levels of mosaicism.

Conclusions: Children with mosaic alleles have fewer tumors per eye compared to those with known high penetrant pathogenic variants and are more likely to remain unilateral. The level of mosaicism has an impact on laterality and number of tumors. 


\section{INTRODUCTION}

Retinoblastoma is the most common paediatric primary ocular cancer and can be heritable. The majority is caused by pathogenic variants (previously known as disease causing mutations) in the RB1 tumor suppressor gene which is located at 13q14. Potentially heritable disease can be divided into 2 groups. One group consists of heterozygous, germline pathogenic variant carriers with the first $R B 1$ allele altered in all cells due to an event during gametogenesis or zygote formation. The second group consists of mosaic RB1 pathogenic variant carriers with 2 or more different genotypes present due to post-zygotic alterations ${ }^{1,2}$. Variant alleles can be further subdivided into alleles associated with high penetrance (HP) and low penetrance (LP). Traditionally the definition and classification of pathogenic variant alleles have been based upon disease eye ratio (DER) for patients with retinoblastoma i.e. the proportion of eyes affected with retinoblastoma ${ }^{3}$. With the development of a clinical classification system ${ }^{4}$ and screening of at-risk patients from birth, it is feasible to quantify the impact of a genetic category according to number of tumors ${ }^{5}$ and also the risk of conversion from unilateral to bilateral disease.

In this study, we compared the number of tumors per eye and conversion from unilateral to bilateral disease in mosaic $R B 1$ pathogenic variant carriers with that of full germline carriers. To add certainty regarding familial patients, patients whose parent carried a pathogenic variant were considered familial full germline carriers: 'de novo' pathogenic variants were not included. 
MATERIALS AND METHODS

114

115 This retrospective study was approved by the Barts Health Clinical Effectiveness

116 Unit (\#7343) and followed the tenets of the Declaration of Helsinki. A retrospective

117 analysis of mosaic and full germline heterozygous $R B 1$ pathogenic variant carriers

118 from 1992 to 2018 was conducted in the Retinoblastoma Genetic Screening Unit

119 (RGSU) at the Royal London Hospital, Barts Health NHS Trust.

121 Genetic Testing

122 Peripheral blood and tumor samples were collected from patients referred to the

123 RGSU for genetic analysis. Consent was obtained from parents/guardians.

124 Techniques used to identify pathogenic variants included conformation analysis,

125 Sanger sequencing, MLPA, QF-PCR and hypermethylation testing as previously

126 described ${ }^{6,7}$. Levels of mosaicism were based upon areas under the peak for

127 sequencing/sizing analysis and titration for standardisation (mixing normal and

128 variant DNA at certain ratios). Levels of mosaicism were defined as high (31-40\%),

129 medium (21-30\%) and low (less than 20\%).

130 The pathogenic variant type was categorised into High Penetrant (HP) or Low

131 Penetrant (LP), and either type could be mosaic. LP variants included promoter,

132 missense and splicing variants (Supplementary tables). Clinical data were collected

133 from notes if available.

134

135 Group Definitions

136 Three groups of patients were included: full germline children with HP alleles, full

137 germline children with LP alleles and children who carried mosaic RB1 pathogenic 
138 variants. To add certainty regarding familial patients, patients whose parent

139 carried a pathogenic variant were considered familial even if they did not manifest

140 the disease: 'de novo' pathogenic variants were not included.

142 In order to ensure all heritable cases were full germline, and not children with high

143 level mosaics, only familial cases were included. It was essential that one of the

144 parents carried a pathogenic variant even if the parent did not manifest the disease.

145 Heterozygous familial cases were screened soon after birth $\left(\mathrm{H} 1^{8}\right)$, but some patients

146 presented with inherited pathogenic variants sporadically at a later age. All children

147 with mosaicism presented sporadically. If unilateral, they were staged as $\mathrm{Hx}$

148 according to the AJCC TNM $8^{\text {th }}$ edition ${ }^{8}$ and converted to $\mathrm{H} 1$ once the molecular

149 testing results were available. Data included age at diagnosis, tumor group

150 according to IIRC ${ }^{4}$, treatment with systemic chemotherapy (for primary treatment or

151 post-enucleation adjuvant chemotherapy: carboplatin, etoposide and vincristine) or

152 external beam radiation (whole eye and lens sparing), number of tumors or foci

153 (including retinomas) per patient and per eye.

155 Number of Tumors

156 The number of tumors was assessed in patients classified with $O, A, B$ and $C$ tumors

157 using the IIRC system: 'gaugeable' eyes. We were keen to assess the number of

158 tumors accurately and not confuse new tumors with subretinal seeds or implants

159 from vitreous seeds. We calculated the total number of tumors in patients with two

160 gaugeable eyes in the 3 groups.

161 As patients often presented with advanced disease in one eye (Groups D or E) and

$162 \mathrm{O} / \mathrm{A} / \mathrm{B} / \mathrm{C}$ (gaugeable) in the other, the number of tumors per eye was recorded in the 
163 gaugeable eye. These eyes were included as tumors per eye. In view of the

164 possibility that there may be a large number of eyes that would never develope a

165 tumor (ie patients staying unilateral), tumor numbers per eye were analysed in 2

166 different ways. (1) in A,B,C eyes that developed tumors excluding eyes that did not

167 manifest disease and (2) in all O,A,B,C eyes including eyes that did not develop

168 tumors.

169

$170 \quad$ Age

171 Only patients with $\mathrm{D}$ or $\mathrm{E}$ group eyes were assessed based upon age at diagnosis as

172 they presented sporadically and not following routine screening of the eyes from birth

173 under general anaesthetic. Laterality of disease was also examined and age of

174 conversion from unilateral to bilateral disease. Patients missing large amounts of

175 data were excluded.

176

177 Statistics

178 A one-tailed Mann-Whitney $U$ test ( $p<0.05$ was deemed statistically significant) was used to determine if there was a significant difference between the three categories:

180 full germline groups (one category of HP variants and the other LP variants) and

181 mosaic patients.

182 The Shapiro-Wilk test was used to mathematically determine whether the data

183 followed a normal distribution. A one-tailed Mann-Whitney $U$ Test $(p<0.05$ was deemed as statistically significant) was used for the comparison of full germline and mosaic patients. Statistical analysis was performed using the Real Statistics

186 Resource Pack ${ }^{9}$, a statistical package add-on for Microsoft Excel (Utah, USA). 
188 RESULTS

189 We identified 137 patients with full germline and mosaic pathogenic variants. After

190 excluding 26 patients (15 full germline and 11 mosaic patients) with insufficient

191 clinical information, data were analysed for 111 patients: 64 were full germline,

192 familial patients (53 HP and 11 LP) as shown in Table 1. 47 were mosaic patients.

193 Figure 1 shows a flow chart demonstrating the selection of the groups.

196 Patient demographics

197 All 64 full germline patients were familial; 59/64 (92\%) had a family history at

198 presentation with an affected parent. Five probands were included who had unaffected carrier parents with a pathogenic variant but were still deemed familial.

200 No children with mosaic disease had a family history and were deemed non-

201 heritable. Proportionally, the gender of patients was similar with $41 \%$ and $40 \%$ of

202 male patients in the full germline and mosaic groups respectively. The remaining

$20359 \%$ and $60 \%$ of both groups were female patients.

204

205 Classification and number of tumors

206 All eyes were classified according to the International Intra-ocular Retinoblastoma

207 Classification and were recorded in Table 1. $85(80 \%)$ of HP eyes, $16(72 \%)$ of LP

208 and $54(57 \%)$ of mosaic eyes had O/A/B/C tumors and were deemed gaugeable

209 such that the number of tumors could be assessed.

210 Total number of tumors in patients with two gaugeable eyes 
211 The total number of tumors was calculated in the 3 groups for patients who had 2

212 eyes staged as $\mathrm{O}, \mathrm{A}, \mathrm{B}$ or $\mathrm{C}$. For 33 patients in the HP group, the median number

213 was 6.0 (mean 6.42 range 1-14) for 2 eyes. For 5 patients in the LP group, the

214 median number was 2.0 (mean 2.8 range 1-5). For 9 patients in the mosaic group,

215 the median number per patient was 1.0 (mean 2.0 range 1-11).

216 Number of tumors per eye for gaugeable eyes

217 1) We assessed the number of tumors per eye in gaugeable eyes that

218 developed new tumors and excluded eyes that never developed tumors, as

219 this reflected clinical experience when parents were keen to know how many

220 more tumors would develop in affected eyes (Table 1). Retinomas were included in this group of tumor foci. 81 (94\%) of gaugeable HP eyes had eyes that developed tumors compared to $8(50 \%)$ of LP eyes and $22(41 \%)$ of mosaics. The number of tumors per eye was fewer in patients with mosaicism (median 1.0 mean 1.9 range 1-6) compared to full germline patients with highly penetrant alleles (median 3.0 mean 3.3 range $1-8)(p<0.000395 \% \mathrm{Cl}$ 0.5, 2.0). Patients in the LP group had a median of 2.0 tumors (mean 2.4 range 1-4) but only 8 eyes were affected.

2) In addition, we evaluated all eyes (including those that never developed a tumor) which were gaugeable. In patients with mosaicism, the median number was 0.0 tumors per eye (mean 0.7 range $0-6$ ) whereas in patients in the HP group, the median number was 3.0 tumors per eye (mean 3.1 range 0-8). Patients with LP pathogenic alleles had a median of 1.0 tumor per eye (mean 1.1 range $0-4$ ) but only 16 eyes were included. 
237 Age at presentation

238 Age at diagnosis was calculated for patients with Group D or E eyes who presented

252 Laterality and age for bilaterality

Presentation with bilateral retinoblastoma was seen in the majority $41 / 53(77 \%)$ of

As the practicality of discussing a median of 0 tumors per eye to parents was questionable, we used different methods to assess tumor numbers. sporadically. 38 of 47 patients (81\%) with mosaicism presented at median age 22 months (range 2-117) compared to 19 of 53 (33\%) of HP patients who presented sporadically at median 7 months (range $0.75-33)(p<0.0000295 \% \mathrm{Cl} 8,21)$. Only 6 of 11 patients with LP had Groups D or E and they presented at median age 27 months (range 12 to 36 months).

Screening under anaesthetic from birth

Despite 59 of 64 full germline cases having a family history at presentation, conventional examination under anaesthesia strategies from birth had been in place for only $29 / 53(55 \%)$ of the HP group, and only $5 / 11$ (45\%) of the LP group were screened. This reflects an earlier era when the screening strategy was being developed. As expected, no child with mosaicism was screened from birth. HP cases in contrast to LP cases with $3 / 11$ (27 \%) and 13/47 (28\%) of mosaic patients. Conversion from unilateral disease to bilateral disease occurred in 8/12 $(67 \%)$ of unilateral HP group cases (median age 5.5 months, mean 6.2, range 3-12) with $49 / 53(92 \%)$ of all cases eventually being bilateral. All eventual bilateral cases 
258 were screened from birth. None of the eight LP group patients with unilateral disease

259 converted to bilateral disease. Only 2 of 34 unilateral patients with mosaicism

260 converted to bilateral disease (mean age 8.5 months, median 8.5, range 8-9). HP

261 patients with unilateral disease were at 11 times increased risk of developing

262 bilateral disease when compared to mosaic patients with unilateral disease (RR

$26311.3,95 \% \mathrm{Cl} 2.8,46.1)$.

265 Level of leukocyte DNA mosaicism and correlation with laterality and number of

266 tumors

267 All patients with mosaicism (32\% bilateral; $68 \%$ unilateral) had pathogenic variants

268 that were deemed HP. Levels of leukocyte mosaicism were classified as low if the

269 variant was less than $20 \%$, medium if $21-30 \%$ and high if $31-40 \%$. Nine of $15(60 \%)$

270 children who had high levels of mosaicism presented as, or became, bilateral.. This

271 compares with only 3 of $22(14 \%)$ patients with low level mosaicism who were

272 bilateral. $7 / 10(70 \%)$ of patients with medium level and 19/22 (86\%) of patients with

273 low level mosaicism were associated with unilateral disease. The number of tumors

274 in affected eyes with mosaicism ranged from 1 to 6 and all 3 children who had more

275 than 2 tumors in one or either eye (unilateral or bilateral) had high levels of

276 mosaicism.

278 Genotype and number of tumors

279 We attempted to assess the number of tumors for the same genotype in either HP or

280 LP groups and compare with the mosaic group. In this cohort of patients, we did not 281 see LP pathogenic variants in any mosaic carriers. Only 3 genotypes (all HP) 
282 overlapped as shown in Table 2. (1) c. 958C>T (exon 10): 11 tumors between 2 eyes

283 in the HP group, but 1 tumor between 2 eyes in the low level mosaic group. (2) c.

$2841654 \mathrm{C}>\mathrm{T}$ (exon 17): 7 tumors between 2 eyes in the HP group, but 1 tumor between

2852 eyes in the medium level mosaic group. (3) c. 2501C>G (exon 24): 5 tumors

286 between 2 eyes in the HP group, but 1 tumor between 2 eyes in the high level

287 mosaic group.

Treatments

291 Patients within this cohort were categorised into no systemic treatment (use of

292 laser/cryotherapy/radioactive plaque/enucleation), intravenous chemotherapy (both

293 primary and post-enucleation adjuvant chemotherapy) and/or external beam

294 radiation therapy (EBRT: both lens-sparing and whole eye). In the HP group,

295 treatment information was available for $46 / 53$ (87\%) patients. 19 of those patients

296 (41\%) had an enucleation and 2 received adjuvant chemotherapy. Altogether 25/46

297 (54\%) had systemic chemotherapy, 14/46 (30\%) had EBRT and only 7/46 (6\%) had

298 neither. 2 patients had both systemic chemotherapy and EBRT. In the LP group, of

29911 patients, six had systemic chemotherapy (55\%), 2 had external beam

300 radiotherapy $(18 \%)$ and $3(27 \%)$ had local treatment throughout. In the mosaic

301 group, 25/47 (53\%) had systemic chemotherapy, 3/47 (6\%) had EBRT and 19/47

302 (40\%) had neither with $18(38 \%)$ having enucleations. 
305 Parents who have suffered from retinoblastoma themselves are keen to know the

306 number of new retinoblastoma tumors that might develop in their children's eyes as

307 soon as they are diagnosed. Parents of children with one eye affected also want to

308 know the chance of bilaterality. This study attempts to address these questions.

309 Hence, we provide prognostic information from the identification of different genetic

310 categories of potentially heritable retinoblastoma.

311 Giving parents information of the number of tumors per eye is practical and useful.

312 This is because parents are often distressed when a tumor develops in a previously

313 unaffected eye and they would like to know how many more might develop. We

314 analysed the data in two ways. When all $\mathrm{O}, \mathrm{A}, \mathrm{B}, \mathrm{C}$ eyes in patients with mosaic $R B 1$

315 alleles were considered, the median number of tumors per eye was 0.0 tumors and

316 we felt this was not meaningful. When only eyes that were affected were included

317 (excluding eyes that did not express disease), the median number of tumors per eye

318 was 1.0 for the mosaic group. We felt this was more useful for parents who had a

319 child with one tumor in one eye and were concerned if more tumors would develop.

320 The median number per eye was 3.0 for HP cases using both analyses.

321 We found that in mosaic carriers, $15 / 47$ (35\%) were or became bilateral.

322 Interestingly, of the 34 mosaic patients with unilateral disease, $32(32 / 34 ; 94 \%)$

323 remained unilateral, which is important information to provide to parents. The two

324 patients who converted from unilateral disease to bilateral disease presented very

325 early (under 3 months of age) with a group $E$ eye and converted 6 months later. No

326 patient with a mosaic pathogenic variant converted to bilateral disease after 9

327 months of age.

328 Genotype-phenotype correlations with respect to the genetic subcategories of HP

329 and LP have been based upon DER as defined by ratio of affected eyes to patients 
330

331

332

333

334

335

336

337

338

339

340

341

342

carrying pathogenic variants. Historically, a disease eye ratio of greater than 1.5 denoted high penetrance disease and less than 1.0 low penetrance ${ }^{3,10}$. With the advent of increased genetic knowledge, the definition of HP and LP are based upon genetic databases ${ }^{1,3}$ rather than DER.

Although one would expect mosaic carriers ${ }^{11,12}$ to be unilateral rather than bilateral and to have an older age at presentation compared to high penetrant disease, this has not been borne out in some previous studies. Rushlow et $a /^{13}$ analysed 45 patients with mosaicism and demonstrated that $23(51 \%)$ were bilateral and only 22 (49\%) were unilateral compared to $28 \%$ and $72 \%$ respectively in this study. Kivela ${ }^{14}$ assessed 13q14 deletions and demonstrated no difference in age and laterality between the 29 mosaics and 107 non-mosaics. However, large deletions including the MED4 gene have a milder non-ocular phenotypic expression ${ }^{15}$ and may behave as LP variants with respect to retinoblastoma. In such cases, the differences will not be clear cut in contrast to HP disease and mosaicism. Neither study assessed the number of tumors. Nor did Rodriguez-Martin et al ${ }^{16}$ whose study showed $14 \%$ of 100 bilateral, and $31 \%$ of 45 unilateral patients displayed mosaicism. However, they reported that mosaicism was associated with late onset retinoblastoma particularly in unilateral patients which we have also found. In addition, our criteria for high level mosaicism $(31 \%-40 \%)$ is below their conservative upper threshold for high level mosaicism $(43 \%)^{16}$ which is reassuring.

We found a correlation between variant percentage in leukocyte DNA and laterality with $60 \%$ (9 of 15) of mosaic patients with bilateral retinoblastoma (at final follow-up) having a high level of mosaic pathogenic variant compared to only $20 \%$ (3 of 15$)$ with low level mosaicism. In addition, all 3 patients (4 eyes) with more than 2 tumors per eye had high level mosaicism. The percentage of white blood cells affected 
355 correlates with the eye involvement and also the number of tumors per eye. Of

356 interest, all pathogenic variants in mosaic carriers were considered as high penetrant

357 pathogenic variants and there was a stark contrast regarding number of tumors

358 between full germline and mosaic carriers (Table 2). There are no reported LP

359 mosaic carriers to our knowledge. It is possible that very low level LP mosaic carriers

360 may not develop the disease due to maintaining sufficient levels of active

361 retinoblastoma protein.

362 In the literature, the number of tumors in affected eyes with familial retinoblastoma

363 has been reported in germline (HP together with LP), with means of $2.19^{17}$ and

$3643.15^{5}$ recorded. Using calculations from original data (Lohmann ${ }^{18}$ et al), we found a

365 mean of 2.8 tumors per eye in HP (nonsense variants) and 2.5 in LP variants (splice

366 site and frameshift). In this study, we found a mean of 3.3 tumors per eye (median

3673.0 ) in HP patients and a mean of 2.4 tumors (median 2.0) in 8 LP eyes and present

368 data for patients carrying mosaic pathogenic variants for the first time.

369 The treatments given may affect the number of tumors formed. New tumor formation

370 has been assessed with systemic chemotherapy for Reese-Ellsworth Groups I to III

371 (equivalent to A, B and C in IIRC or cT1 and CT2a in the AJCC) ${ }^{19}$ :for seven patients,

37236 new tumors developed in 11 eyes (mean 3.2). This is comparable to the mean of

3733.3 tumors per eye in HP patients noted in this study with different treatment

374 modalities. It has been suggested that systemic chemotherapy may delay the onset

375 of new tumors, but ethically it is difficult to conduct a comparative trial to prove this.

376 We had similar proportions of HP, LP and mosaic patients who had systemic

377 chemotherapy (53-55\%). Similarly for EBRT, new tumor development can be

378 retarded but comparison with purely local treatment groups has proven difficult ${ }^{20,21}$. 
379 Giving figures related to conversion of unilateral disease to bilateral disease is useful

380 for families. Only a small proportion (6\%) of mosaic patients converted from

381 unilateral to bilateral disease and this may be related to the older age of non-

382 screening (sporadic) presentation compared to the majority of germline patients who

383 were screened from birth. But only $32 \%$ (15/47) of mosaics were eventually bilateral

384 compared to $92 \%(49 / 53)$ of HP patients. Although we did not find any child who

385 converted from unilateral to bilateral disease after the age of 12 months, Temming et

$386 a^{22}$ noted 3 patients who converted to bilateral disease after this age in their 1961-

3872006 cohort.

388 Next generation sequencing is better able to detect low level mosaics ${ }^{16}$ and future

389 studies may be able to delineate these findings more accurately.

390 Limitations

391 We assessed LP patients for the number of tumors but we had data for only 8 eyes

392 with gaugeable eyes and tumor development.It is difficult to make conclusions based

393 upon this limited sample size. We had insufficient clinical information for 26 patients

394 which may have affected results.

395 Treatment may have had an impact on the number of tumors per eye recorded. We

396 limited our assessment of number of tumors to only eyes without substantial

397 subretinal and vitreous seeding which reduced the number of eyes being assessed.

398 Systemic chemotherapy and temporal approach EBRT (including lens sparing) may

399 have treated the eye with the more aggressive disease, but also the fellow eye

400 without disease. Similar proportions of HP and mosaic patients had systemic

401 chemotherapy. We only had 7 patients in the HP group who had neither treatment. 
402 No patients had first line intra-arterial chemotherapy, which can be systemically

403 absorbed and may have an impact on both eyes despite being given to one eye.

404 We may have been unable to detect low level mosaics with unilateral disease and

405 instead labelled them as having non-heritable somatic pathogenic variants (and

406 excluded them from this study) due to the limitations of technology used. We are

407 reassured as we screened the offspring of the patients via examinations under

408 anaesthesia and did not find any affected. However, this is not completely

409 confirmatory.

410 Conclusions

411 In summary, this is the first study to demonstrate increased unilateral disease (rather

412 than bilateral) and fewer tumors per eye for mosaicism compared to high penetrant

413 disease in retinoblastoma. The expected number of tumor foci in patients with

414 somatic mosaicism is lower compared to full germline patients heterozygous for the

415 same variant $R B 1$ allele. Details regarding number of tumors can be provided to

416 parents/guardians for prognostic information for different categories of potentially

417 heritable retinoblastoma

418

419

420

421

422

423

424

425

426

427

428

429 
REFERENCES

431

432 1. Soliman SE, Racher H, Zhang C, et al. Genetics and Molecular Diagnostics in Retinoblastoma--An 433 Update. Asia Pac J of Ophthalmol. 2017;6:197-207.

434 2. Sippel KC, Fraioli RE, Smith GD, et al. Frequency of somatic and germ-line mosaicism in

435 retinoblastoma: implications for genetic counseling. Am J Hum Genet. 1998;62:610-619.

436 3. Lohmann DR, Brandt B, Hopping W, et al. Distinct RB1 gene mutations with low penetrance in

437 hereditary retinoblastoma. Hum Genet. 1994;94:349-354.

438 4. Murphree L. Intraocular Retinoblastoma: the case for a new group classification. Ophthalmol Clin 439 North Am. 2005;18:41-53.

440 5. Munier FL, Balmer A, van Melle G, et al. Radial asymmetry in the topography of retinoblastoma.

441 Clues to the cell of origin. Ophthalmic Genet. 1994;15:101-106.

442 6. Price EA, Price K, Kolkiewicz K, et al. Spectrum of RB1 mutations identified in 403 retinoblastoma 443 patients. J Med Genet. 2014;51:208-214.

444 7. Price EA, Kolkiewicz K, Patel R, et al. Detection and reporting of RB1 promoter hypermethylation in 445 diagnostic screening. Ophthalmic Genet. 2018;39:526-531.

446 8. Mallipatna AC, Gallie BL, Chevez-Barrios P, et alRetinoblastoma. In Amin MB, Edge SB, Greene 447 FL,et al, eds . AJCC Cancer Staging Manual. 8th edNew York: Springer; 2017:819-831.

448 9. Zaiontz C. Real Statistics Resource Pack. Beta-for Macintosh. http:/www.real-statistics.com/free449 download/real-statistics-resource-pack/real-statistics-resource-pack-macintosh/comment-page-1/; 2019

450 Accessed 21.03.20

451 10. Taylor M, Dehainault C, Desjardins L, et al. Genotype-phenotype correlations in hereditary familial 452 retinoblastoma. Hum Mutat. 2007;28:284-293.

453 11. Carlson EA, Desnick RJ. Mutational mosaicism and genetic counseling in retinoblastoma. Am J Med 454 Genet. 1979;4:365-381.

455 12. Munier FL, Beck-Popovic M, Chantada GL, et al. Conservative management of retinoblastoma:

456 Challenging orthodoxy without compromising the state of metastatic grace. "Alive, with good vision and no 457 comorbidity". Prog Retin Eye Res. 2019;73:10076.

458 13. Rushlow D, Piovesan B, Zhang K, et al. Detection of mosaic RB1 mutations in families with 459 retinoblastoma. Hum Mutat. 2009;30:842-851.

460 14. Kivela T, Tuppurainen K, Riikonen P, et al. Retinoblastoma associated with chromosomal $13 \mathrm{q} 14$

461 deletion mosaicism. Ophthalmology. 2003;110:1983-1988.

462 15. Mitter D, Ullmann R, Muradyan A, et al. Genotype-phenotype correlations in patients with 463 retinoblastoma and interstitial 13q deletions. Eur J Hum Genet. 2011;19:947-958.

464 16. Rodríguez-Martín C, Robledo C, Gómez-Mariano G, et al. Frequency of low-level and high-level 465 mosaicism in sporadic retinoblastoma: genotype-phenotype relationships. J Hum Genet. 2020;65:165-174.

466 17. King BA, Parra C, Li Y, et al. Spatiotemporal Patterns of Tumor Occurrence in Children with

467 Intraocular Retinoblastoma. PloS One. 2015;10:e0132932. 
468 18. Lohmann DR, Brandt B, Hopping W, et al. The spectrum of RB1 germ-line mutations in hereditary

469 retinoblastoma. Am J Hum Genet. 1996;58:940-949.

470 19. Wilson MW, Haik BG, Billups CA, et al. Incidence of new tumor formation in patients with hereditary 471 retinoblastoma treated with primary systemic chemotherapy: is there a preventive effect? Ophthalmology.

$472 \quad 2007 ; 114: 2077-2082$.

473 20. Messmer EP, Sauerwein W, Heinrich T, et al. New and recurrent tumor foci following local treatment 474 as well as external beam radiation in eyes of patients with hereditary retinoblastoma. Graefes Arch. Clin.Exp. 475 Ophthalmol.. 1990;228:426-431.

476 21. Roysarkar TK, Biswas J, Gopal L. New tumours in non-enucleated eyes of bilateral retinoblastoma 477 patients. Indian J Ophthalmol. 1994;42:19-22.

478 22. Temming P, Viehmann A, Biewald E, et al. Sporadic unilateral retinoblastoma or first sign of bilateral 479 disease? Br J Ophthalmol. 2013;97:475-480. 
483

484

485

486

487

Figure 1. A flow chart demonstrating the selection of the groups

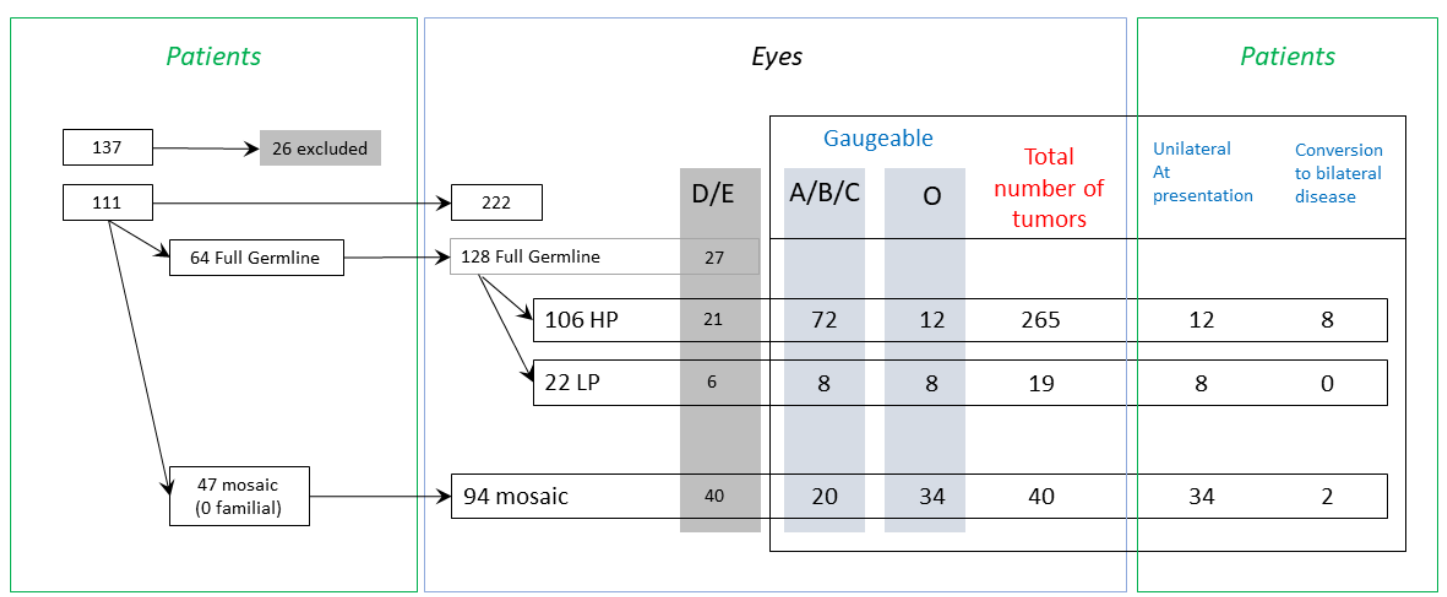

488

489

490 
491 Table 1. Characteristics for genetic subtypes of $R B 1$ pathogenic variants

\begin{tabular}{|c|c|c|c|}
\hline & $\mathrm{HP}$ & LP & Mosaic \\
\hline Patients (eyes) & $53(106)$ & $11(22)$ & $47(94)$ \\
\hline Group 0 & $12(12 \%)$ & $8(36 \%)$ & $34(36 \%)$ \\
\hline Group A-C & $72(68 \%)$ & $8(36 \%)$ & $20(21 \%)^{*}$ \\
\hline Group D-E & $21(20 \%)$ & $6(28 \%)$ & $40(43 \%)$ \\
\hline $\begin{array}{l}\text { Gaugeable eyes } \\
\text { that developed } \\
\text { tumors }\end{array}$ & 81 & 8 & 22 \\
\hline $\begin{array}{l}\text { No of Tumors } \\
\text { per eye ( affected } \\
\text { eyes only) } \\
\text { Median } \\
\text { (mean,range) }\end{array}$ & $3.0(3.9,1-7)$ & $2.0(2.4,1-4)$ & $1.0(1.9,1-6)$ \\
\hline $\begin{array}{l}\text { Age (months) at } \\
\text { diagnosis for } \\
\text { sporadic cases } \\
\text { Median (mean, } \\
\text { range) }\end{array}$ & $\begin{array}{l}7.00(8.42,0.75- \\
33)\end{array}$ & $\begin{array}{l}27.00 \\
(25.00,12-36)\end{array}$ & $\begin{array}{l}21.00 \\
(25.11,2-117)\end{array}$ \\
\hline \multicolumn{4}{|l|}{ Management } \\
\hline $\begin{array}{l}\text { Screened under } \\
\text { anaesthetic }\end{array}$ & $29(55 \%)$ & $5(45 \%)$ & 0 \\
\hline $\begin{array}{l}\text { Systemic } \\
\text { Chemotherapy }\end{array}$ & 21 & 4 & 22 \\
\hline Radiotherapy & 12 & 2 & 3 \\
\hline Both & 6 & 1 & 1 \\
\hline None & 5 & 3 & 20 \\
\hline $\begin{array}{l}\text { Incomplete } \\
\text { Information }\end{array}$ & 9 & 1 & 1 \\
\hline Enucleation & $19(36 \%)$ & 6 & $37(79 \%)$ \\
\hline $\begin{array}{l}\text { Unilateral at } \\
\text { presentation }\end{array}$ & 12 & 8 & 34 \\
\hline Stayed unilateral & $4(33 \%)$ & 8 & $32(94 \%)$ \\
\hline Became bilateral & 8 & 0 & 2 \\
\hline
\end{tabular}

493 *including 2 eyes with retinomas 
494

495

496

497

498

499
Table 2. RB1 genotypes present in both the full germline and mosaic groups

\begin{tabular}{|l|l|l|}
\hline RB1 Nonsense Variant & Full germline HP group & Mosaic group \\
(both eyes) & (both eyes) \\
\hline c. $958 \mathrm{C}>$ T exon 10 & 11 tumours & 1 tumour (low level) \\
\hline c. $1654 \mathrm{C}>$ T exon 17 & 7 tumours & 1 tumour (medium level) \\
\hline c. $2501 \mathrm{C}>$ G exon 24 & 5 tumours & 1 tumour (high level) \\
\hline
\end{tabular}


500 Supplementary Tables 


\begin{tabular}{|c|c|c|c|c|c|c|c|}
\hline Presentation & $\begin{array}{l}\text { Age at } \\
\text { diagnosis } \\
\text { (m) }\end{array}$ & RB1 g. no. L11910.1 & RB1 c. no. LRG_517t1 (RB1) & RB1 ex/int/pro & $\begin{array}{l}\text { Putative } \\
\text { consequence }\end{array}$ & $\begin{array}{l}\text { Final } \\
\text { laterality } \\
\text { (U/B) }\end{array}$ & $\begin{array}{l}\text { Tumour } \\
\text { noleye }\end{array}$ \\
\hline$A / A$ & 0.50 & & c.(?_-166)_(264+1_265-1)del & pro-int2 del & expression & B & 3 \\
\hline $\mathrm{A} / \mathrm{A}$ & 0.50 & g. $162298 C>A$ & c. $2420 \mathrm{C}>\mathrm{A}$ & ex23 & p.(S807*) & $\mathrm{B}$ & 2.5 \\
\hline$A / A$ & 1.50 & $\operatorname{del}(13)(q 14.1 q 21)$ & & delRB1x1 & no $p R b$ & B & 2 \\
\hline $\mathrm{A} / \mathrm{A}$ & 3.00 & & $\begin{array}{l}\text { c. }\left(1215+1 \_1216-\right. \\
\text { 1)_(1332+1_1333-1)del }\end{array}$ & ex13 del & p. $\left(406 \_444\right) \mathrm{del}$ & $\mathrm{B}$ & 2.5 \\
\hline$A / A$ & 3.00 & g.73801dupA & c.1264dupA & ex13 & p. $(1422 \mathrm{Nfs} * 6)$ & $\mathrm{B}$ & 3.5 \\
\hline$A / B$ & 0.25 & & c.(?_-166)_(264+1_265-1)del & pro-int2 del & expression & $\mathrm{B}$ & 7 \\
\hline$A / B$ & 0.25 & g. $64348 \mathrm{C}>\mathrm{T}$ & c.958C $>\mathrm{T}$ & ex10 & p. $($ R320*) & $\mathrm{B}$ & 5.5 \\
\hline$A / B$ & 1.00 & & c.(1695+1_1696-1)_(*1815_?)del & ex18-beyond 3'del & no pRb & $\mathrm{B}$ & 2.5 \\
\hline$A / B$ & 1.00 & g. $170383 C>G$ & c. $2501 C>G$ & ex24 & p.(S834*) & $\mathrm{B}$ & 2.5 \\
\hline$A / B$ & 1.25 & g.59759_59778del20 & c.827_846del20 & ex8 & p. $\left(\mathrm{L} 277^{*}\right)$ & $\mathrm{B}$ & 3 \\
\hline$A / B$ & 3.00 & & c. $\left(1695+1 \_1696-1\right) \_\left({ }^{*} 1815 \_?\right) \mathrm{del}$ & ex18-beyond 3'del & no $p R b$ & $\mathrm{~B}$ & 1.5 \\
\hline$A / B$ & 4.00 & g.56,963-56,964insAT & c.718_719insAT & ex7 & p. $\left({\left.\mathrm{K} 240 N f s^{*} 25\right) / \text { splice }}\right.$ & $\mathrm{B}$ & 1.5 \\
\hline$A / D$ & 7.00 & g.59683C >T & c. $751 \mathrm{C}>\mathrm{T}$ & ex8 & p. $\left(\mathrm{R} 251^{*}\right)$ & $\mathrm{B}$ & 3 \\
\hline $\mathrm{A} / \mathrm{O}$ & 0.50 & g.45867G $>\mathrm{T}$ & c. $607+1 \mathrm{G}>\mathrm{T}$ & int6 & $\begin{array}{l}\text { sd/ex } 6 \mathrm{skip} / \\
\text { p.(I181Gf*8) }\end{array}$ & $\mathrm{B}$ & 1.5 \\
\hline $\mathrm{A} / \mathrm{O}$ & 0.50 & g.45867G >C & c. $607+1 G>C$ & int6 & $\begin{array}{l}\text { sd/ex } 6 \mathrm{skip} / \\
\text { p.(I181Gfs*8) }\end{array}$ & $\mathrm{B}$ & 3 \\
\hline$A / A$ & 1.00 & g. $162112 T>G$ & c. $2325+2 T>G$ & int22 & sd & $\mathrm{B}$ & 5.5 \\
\hline $\mathrm{B} / \mathrm{A}$ & 0.25 & g.150062_150071del10 & c.1760_1769del10 & ex18 & p.(E587Vfs*21) & $\mathrm{B}$ & 1 \\
\hline $\mathrm{B} / \mathrm{A}$ & 1.00 & g.56862T>A & c.617T $>A$ & ex7 & p. $($ L206*) & $\mathrm{B}$ & 2.5 \\
\hline $\mathrm{B} / \mathrm{A}$ & 10.00 & g.2104_2135del32 & c.45-76del32 & ex1 & p.(A17Pfs*3) & $\mathrm{B}$ & 1 \\
\hline $\mathrm{B} / \mathrm{B}$ & 0.25 & g.39478G>A & c. $297 \mathrm{G}>\mathrm{A}$ & ex3 & p.(W99*) & $\mathrm{B}$ & 2 \\
\hline $\mathrm{B} / \mathrm{B}$ & 0.75 & g.41954G>T & c.409G $>\mathrm{T}$ & ex4 & p. $\left(\mathrm{E} 137^{*}\right)$ & $\mathrm{B}$ & 4.5 \\
\hline $\mathrm{B} / \mathrm{B}$ & 1.50 & & c. $\left(1695+1 \_1696-1\right) \_\left({ }^{*} 1815 \_?\right) \mathrm{del}$ & ex18-beyond 3'del & no $\mathrm{pRb}$ & $\mathrm{B}$ & 5.5 \\
\hline $\mathrm{B} / \mathrm{B}$ & 1.00 & g. $77080 A>T$ & c. $1498+3 A>T$ & int16 & sd & $\mathrm{B}$ & 1.5 \\
\hline $\mathrm{B} / \mathrm{B}$ & 6.00 & g. $70240 A>G$ & c. $1128-2 A>G$ & int11 & sa & $\mathrm{B}$ & 3.5 \\
\hline $\mathrm{B} / \mathrm{B}$ & 6.00 & g. $39562 \mathrm{G}>\mathrm{T}$ & c. $380+1 G>T$ & int3 & $\begin{array}{l}\text { sd/ex } 3 \mathrm{skip} / \\
\text { p.(G89Cfs*3) }\end{array}$ & $\mathrm{B}$ & 3.5 \\
\hline $\mathrm{D} / \mathrm{O}$ & 2.00 & & c.(?_-166)_(*1815_?)del & delRB1x1 & no $\mathrm{pRb}$ & $\mathrm{B}$ & 2 \\
\hline$B / D$ & 0.75 & g.42018T>A & c. $473 T>A$ & ex4 & p. $($ L158*) & $\mathrm{B}$ & 4 \\
\hline
\end{tabular}




\begin{tabular}{|c|c|c|c|c|c|c|c|}
\hline$B / D$ & 9.00 & g.45867G >C & c. $607+1 \mathrm{G}>\mathrm{C}$ & int6 & $\begin{array}{l}\text { sd/ex } 6 \mathrm{skip} / \\
\text { p.(I181Gfs*8) }\end{array}$ & B & 1 \\
\hline $\mathrm{B} / \mathrm{E}$ & 2.00 & g. $76460 \mathrm{C}>\mathrm{T}$ & c. $1363 C>T$ & ex14 & p. $\left(\mathrm{R} 455^{*}\right)$ & $\mathrm{B}$ & 2 \\
\hline$B / E$ & 24.00 & g.162364C>A & c. $2486 C>A$ & ex23 & p.(S829*) & $\mathrm{B}$ & 7 \\
\hline $\mathrm{B} / \mathrm{O}$ & 0.50 & g.45844G>A & c. $585 \mathrm{G}>\mathrm{A}$ & ex6 & p. $($ W195*) & B & 3.5 \\
\hline $\mathrm{B} / \mathrm{O}$ & 1.00 & g. $64348 \mathrm{C}>\mathrm{T}$ & c. $958 \mathrm{C}>\mathrm{T}$ & ex10 & p.(R320*) & $\mathrm{B}$ & 2.5 \\
\hline $\mathrm{B} / \mathrm{O}$ & 7.00 & & c. $\left.\left(1695+1 \_1696-1\right) \_{ }^{*} 1815 \_?\right)$ del & ex18-beyond 3'del & no $\mathrm{pRb}$ & $U$ & 1.5 \\
\hline $\mathrm{C} / \mathrm{B}$ & 10.00 & g.76894delA & c.1395delA & ex15 & p.(E466Nfs*12) & $\mathrm{B}$ & 5.5 \\
\hline$B / B$ & 2.00 & g. $78238 \mathrm{C}>\mathrm{T}$ & c. $1654 C>T$ & ex17 & g.(R552*) & $B$ & 3.5 \\
\hline $\mathrm{C} / \mathrm{C}$ & 4.00 & g. $61733 \mathrm{~A}>\mathrm{T}$ & c. $865 \mathrm{~A}>\mathrm{T}$ & ex9 & p. $(\mathrm{K} 289 *)$ & $\mathrm{B}$ & 5 \\
\hline$D / D$ & 5.00 & g.70330G >A & c. $1215+1 G>A$ & int12 & $\begin{array}{l}\text { sd/ex } 12 \mathrm{skip} / \\
\text { p.(V378Afs*3) }\end{array}$ & B & NA \\
\hline$C / D$ & 9.00 & g. $64348 \mathrm{C}>\mathrm{T}$ & c. $958 \mathrm{C}>\mathrm{T}$ & ex10 & p. $\left(\mathrm{R} 320^{*}\right)$ & B & 5 \\
\hline $\mathrm{C} / \mathrm{E}$ & 1.00 & g.39445G $>A$ & c. $265-1 G>A$ & int2 & sa & $\mathrm{B}$ & 6 \\
\hline $\mathrm{C} / \mathrm{O}$ & 1.25 & g.70330G >A & c. $1215+1 G>A$ & int12 & $\begin{array}{l}\text { sd/ex } 12 \text { skip/ } \\
\text { p.(V378Afs*3) }\end{array}$ & B & 5 \\
\hline $\mathrm{C} / \mathrm{O}$ & 36.00 & g.150050delC & c.1748delC & ex18 & p. $(\mathrm{T} 583 \mathrm{Mfs} * 28)$ & $U$ & 0.5 \\
\hline $\mathrm{D} / \mathrm{B}$ & 1.25 & g.170405_170408delGAGT & c. $2520+3 \_2520+6$ delGAGT & int24 & $\begin{array}{l}\text { sd/ex } 24 \text { skip/ } \\
\text { (p.1831Lfs } 8)\end{array}$ & B & 4 \\
\hline $\mathrm{D} / \mathrm{B}$ & 9.00 & g.77078G $>\mathrm{T}$ & c. $1498+1 G>T$ & int16 & sd & B & 1 \\
\hline $\mathrm{D} / \mathrm{C}$ & 4.00 & $\begin{array}{l}\text { g.153354_153359delGTTAGTins } \\
22\end{array}$ & c.1960+1_1960+6delGTTAGTins22 & int19 & $\begin{array}{l}\text { sd/ex } 19 \mathrm{skip} / \\
\text { p.(M605lfs*14) }\end{array}$ & $\mathrm{B}$ & 5 \\
\hline $\mathrm{D} / \mathrm{C}$ & 33.00 & g.2079delG & c.20delG & ex1 & p.(R7Qfs*58) & $B$ & 1 \\
\hline$E / B$ & 2.00 & g.59646_59649delTACAins18 & c.719-5_719-2delTACAins18 & int7 & sa & B & 6 \\
\hline$E / B$ & 7.00 & g.73809_73818dup10 & c.1272_1281dup10 & ex13 & p.(E428Hfs*3) & B & 4 \\
\hline $\mathrm{D} / \mathrm{D}$ & 11.00 & g.162237C >T & c. $2359 C>T$ & ex23 & p. $($ R787*) & B & NA \\
\hline $\mathrm{O} / \mathrm{D}$ & 13.00 & g.162237C $>T$ & c. $2359 \mathrm{C}>\mathrm{T}$ & ex23 & p. $\left(\mathrm{R} 787^{*}\right)$ & $U$ & NA \\
\hline$E / O$ & 16.00 & g.45867G>T & c. $607+1 \mathrm{G}>\mathrm{T}$ & int6 & $\begin{array}{l}\text { sd/ex } 6 \text { skip/ } \\
\left.\text { p.(I181 } \mathrm{Gs}^{*} 8\right)\end{array}$ & $U$ & NA \\
\hline$E / C$ & 4.00 & g. $76460 C>T$ & c. $1363 \mathrm{C}>\mathrm{T}$ & ex14 & p.(R455*) & $\mathrm{B}$ & 2 \\
\hline $\mathrm{O} / \mathrm{A}$ & 2.00 & g.149997G>A & c.1696-1G>A & int17 & sa & $\mathrm{B}$ & 5.5 \\
\hline
\end{tabular}


502

Table 2a. Pathogenic variant data for High Penetrant $R B 1$ variant patients.

Genomic (g.) nucleotide numbering is according to GenBank sequence accession number L11910.1. In cDNA (c.) nucleotide numbering c.1 is the $A$ of the ATG translation initiation codon based on the Locus Reference Genomic Sequence LRG_517t1 (RB1). Variant nomenclature is according to Human Genome Variation Society guidelines (www.hgvs.org). ex - exon, int- intron, pro- promoter, sd- splice donor, sa- splice acceptor, g- germline, U- unilateral, B- bilateral. NA- not applicable

\begin{tabular}{|c|c|c|c|c|c|c|c|}
\hline Presentation & $\begin{array}{l}\text { Age at } \\
\text { diagnosis } \\
\text { (m) }\end{array}$ & RB1 g. no. L11910.1 & $\begin{array}{l}\text { RB1 c. no. } \\
\text { LRG_517t1 (RB1) }\end{array}$ & $\begin{array}{l}R B 1 \\
\text { ex/int/pro }\end{array}$ & Putative consequence & $\begin{array}{l}\text { Final } \\
\text { laterality(U/B) }\end{array}$ & Tumour noleye \\
\hline B/B & 0.25 & g.160834G >C & c. $2211 G>C$ & ex21 & p.(E737D)/sd & B & 2.5 \\
\hline$A / D$ & 12 & g.45867G $>T$ & c. $607+1 \mathrm{G}>\mathrm{T}$ & int6 & $\begin{array}{l}\text { sd/ex } 6 \\
\text { skip/p.(1181Gfs*8) }\end{array}$ & B & 2 \\
\hline $\mathrm{B} / \mathrm{O}$ & 14 & g.2104_2135del32 & c.45_76del32 & ex1 & p.(A17Pfs*3) & $U$ & 2 \\
\hline $\mathrm{B} / \mathrm{O}$ & 14 & g.1867T>A & c. $-193 T>A$ & pro & expression & $U$ & 0.5 \\
\hline
\end{tabular}


521

\begin{tabular}{|c|c|c|c|c|c|c|c|}
\hline$E / O$ & 20 & g. $156713 C>T$ & c. $1981 C>T$ & ex20 & p.(R661W) & U & NA \\
\hline$B / O$ & 32 & g.65378_65379delGA & c.1064_1065delGA & ex11 & p.(R355Nfs*6) & $U$ & 1 \\
\hline $\mathrm{E} / \mathrm{O}$ & 34 & g.59793G >A & c. $861 \mathrm{G}>\mathrm{A}$ & ex8 & p. $(E 287=) / s d$ & $U$ & NA \\
\hline
\end{tabular}

Table 2b. Pathogenic variant data for Low Penetrant $R B 1$ variant patients.

Genomic (g.) nucleotide numbering is according to GenBank sequence accession number L11910.1. In cDNA (c.) nucleotide numbering c.1 is the A of the ATG translation initiation codon based on the Locus Reference Genomic Sequence LRG_517t1 (RB1). Varaint nomenclature is according to Human Genome Variation Society guidelines (www.hgvs.org). ex - exon, int- intron, pro- promoter, sd- splice donor, sa- splice acceptor, g- germline, U- unilateral, B- bilateral. NA- not applicable 


\begin{tabular}{|c|c|c|c|c|c|c|c|c|}
\hline & & & 27 & & & & & \\
\hline $\mathrm{E} / \mathrm{O}$ & 2 & g.59794G >A & c. $861+1 G>A$ & int8 & sd & $\mathrm{B}$ & 1 & Medium \\
\hline $\mathrm{O} / \mathrm{E}$ & 3 & g.150117G >C & c. $1814+1 G>C$ & int18 & sd & $\mathrm{B}$ & 4 & High \\
\hline $\mathrm{B} / \mathrm{C}$ & 7 & g.162317T>G & c. $2439 \mathrm{~T}>\mathrm{G}$ & ex23 & p. $\left(Y_{813^{*}}\right)$ & $\mathrm{B}$ & 5.5 & High \\
\hline $\mathrm{A} / \mathrm{C}$ & 8 & g.73843C>T & c. $1306 \mathrm{C}>\mathrm{T}$ & ex13 & p. $\left(\mathrm{Q} 436^{*}\right)$ & $B$ & 1.5 & Low \\
\hline $\mathrm{D} / \mathrm{C}$ & 9 & g. $150037 \mathrm{C}>\mathrm{T}$ & c. $1735 \mathrm{C}>\mathrm{T}$ & ex18 & p. $($ R579*) & $\mathrm{B}$ & 2 & Medium \\
\hline $\mathrm{O} / \mathrm{D}$ & 9 & g.2121delC & c.62delC & ex1 & p. $\left(\mathrm{P} 21 \mathrm{Rfs}^{*} 44\right)$ & $\mathrm{U}$ & NA & Low \\
\hline $\mathrm{O} / \mathrm{D}$ & 20 & g.65363G $>A$ & c. $1050-1 G>A$ & int10 & sa & $\mathrm{U}$ & NA & Low \\
\hline $\mathrm{E} / \mathrm{O}$ & 9 & g.76898C>T & c. $1399 \mathrm{C}>\mathrm{T}$ & ex15 & p. $\left(\mathrm{R} 467^{*}\right)$ & $\mathrm{U}$ & NA & Medium \\
\hline $\mathrm{A} / \mathrm{E}$ & 10 & g.78217G >T & c. $1633 G>T$ & ex17 & p. $($ E545*) & $B$ & 1 & Medium \\
\hline $\mathrm{D} / \mathrm{O}$ & 10 & g.76430C $>T$ & c. $1333 \mathrm{C}>\mathrm{T}$ & ex14 & p. $\left(\mathrm{R} 445^{*}\right)$ & $U$ & NA & Low \\
\hline $\mathrm{E} / \mathrm{O}$ & 10 & g.156774G >A & c. $2042 G>A$ & ex20 & p. $\left(\mathrm{W} 681^{*}\right)$ & $\mathrm{U}$ & NA & Low \\
\hline $\mathrm{D} / \mathrm{C}$ & 11 & g.70298_71084delinsTG & c. $1184 \_1215+755$ delinsTG & ex12 & $\begin{array}{l}\begin{array}{l}\text { p.(Q395_N405 } \\
\text { delins43) }\end{array} \\
\end{array}$ & $\mathrm{B}$ & 2 & High \\
\hline $\mathrm{O} / \mathrm{C}$ & 11 & g.78,152_78,155dupTAAA & c.1568_1571dupTAAA & ex17 & p. $\left(\mathrm{K} 524 \mathrm{Nfs}{ }^{*} 5\right)$ & $\mathrm{U}$ & 0.5 & Medium \\
\hline $\mathrm{O} / \mathrm{D}$ & 11 & & c. $(?-166)\left(* 1815 \_?\right) \mathrm{del}$ & delRB1x1 & no $\mathrm{pRb}$ & $\mathrm{U}$ & NA & High \\
\hline $\mathrm{D} / \mathrm{O}$ & 11 & & c. $(?-166) \_\left({ }^{*} 1815 \_?\right) \mathrm{del}$ & delRB1x1 & no $p R b$ & U & NA & Low \\
\hline $\mathrm{C} / \mathrm{O}$ & 11 & g.78238C>T & c. $1654 \mathrm{C}>\mathrm{T}$ & ex17 & p. $($ R552*) & U & 0.5 & Medium \\
\hline $\mathrm{O} / \mathrm{D}$ & 12 & g.153352dupA & c.1959dupA & ex19 & p.(V654Sfs*14) & $\mathrm{U}$ & NA & Medium \\
\hline $\mathrm{D} / \mathrm{E}$ & 12 & g.76921G $>C$ & c. $1421+1 \mathrm{G}>\mathrm{C}$ & int15 & sd & $\mathrm{B}$ & NA & High \\
\hline $\mathrm{E} / \mathrm{B}$ & 13 & g.70004_70672del & c.1128-238_1215+343del & ex 12 skip & p.(V378Afs*3) & $\mathrm{B}$ & 2 & High \\
\hline $\mathrm{O} / \mathrm{E}$ & 13 & g.56,903-56,909del7 & c.658_664del7 & ex7 & p. (L220Sfs*42) & $U$ & NA & High \\
\hline O/D & 14 & g.76910C>T & c. $1411 \mathrm{C}>\mathrm{T}$ & ex15 & p. $\left(\mathrm{Q} 471^{*}\right)$ & $\mathrm{U}$ & NA & Low \\
\hline $\mathrm{O} / \mathrm{D}$ & 18 & g.64348C>T & c. $958 \mathrm{C}>\mathrm{T}$ & ex10 & p. $\left(\mathrm{R} 320^{*}\right)$ & $\mathrm{U}$ & NA & Low \\
\hline $\mathrm{O} / \mathrm{D}$ & 20 & g.150037C>T & c. $1735 \mathrm{C}>\mathrm{T}$ & ex18 & p.(R579*) & $\mathrm{U}$ & NA & Low \\
\hline$E / B$ & 22 & g.156785C>T & c. $2053 \mathrm{C}>\mathrm{T}$ & ex20 & p. $($ Q685*) & $\mathrm{B}$ & 1 & Low \\
\hline $\mathrm{D} / \mathrm{B}$ & 24 & g.70280T>A & c. $1166 \mathrm{~T}>\mathrm{A}$ & ex12 & p.(L389*) & $B$ & 5 & High \\
\hline O/D & 24 & g.76975_77081del107 & c.1422-26_1498+4del107 & int15_int16 & p.(S474Rfs*) & $U$ & NA & Low \\
\hline $\mathrm{O} / \mathrm{D}$ & 25 & g.59695C $>T$ & c. $763 \mathrm{C}>\mathrm{T}$ & ex8 & p. $\left(\mathrm{R} 255^{\star}\right)$ & $\mathrm{U}$ & NA & Medium \\
\hline $\mathrm{O} / \mathrm{C}$ & 25 & g.64348C>T & c. $958 \mathrm{C}>\mathrm{T}$ & ex10 & p. $\left(\mathrm{R} 320^{*}\right)$ & U & 0.5 & Low \\
\hline $\mathrm{C} / \mathrm{O}$ & 27 & & c. $\left(1695+1 \_1696-1\right) \_\left({ }^{*} 1815 \_?\right) \mathrm{del}$ & $\begin{array}{l}\text { ex18-beyond } \\
\text { 3'del }\end{array}$ & no $\mathrm{pRb}$ & $U$ & 0.5 & High \\
\hline $\mathrm{A} / \mathrm{E}$ & 28 & g.153352delA & c.1959delA & ex19 & p.(V654Cfs*4) & $\mathrm{B}$ & 1 & High \\
\hline $\mathrm{D} / \mathrm{O}$ & 28 & g.76460C>T & c. $1363 \mathrm{C}>\mathrm{T}$ & ex14 & p. $\left(\mathrm{R} 455^{*}\right)$ & $U$ & NA & Medium \\
\hline $\mathrm{E} / \mathrm{O}$ & 30 & g.78250C>T & c. $1666 \mathrm{C}>\mathrm{T}$ & ex17 & p.(R556*) & $\mathrm{U}$ & NA & Low \\
\hline $\mathrm{O} / \mathrm{B}$ & 30 & g. $170383 C>G$ & c. $2501 \mathrm{C}>\mathrm{G}$ & ex24 & p. $\left(\mathrm{S} 834^{*}\right)$ & $U$ & 0.5 & High \\
\hline
\end{tabular}




\begin{tabular}{|c|c|c|c|c|c|c|c|c|}
\hline $\mathrm{O} / \mathrm{E}$ & 54 & g.156785C>T & c. $2053 C>T$ & ex20 & p. $\left(Q 685^{*}\right)$ & $\mathrm{U}$ & NA & Low \\
\hline $\mathrm{O} / \mathrm{D}$ & 43 & g.76932_76952del21 & c. $1421+12 \_1421+32$ del 21 & int15 & sd & $\mathrm{U}$ & NA & Low \\
\hline $\mathrm{D} / \mathrm{O}$ & 43 & delint23_int26 & c. $\left.2489+1 \_2490-1\right) \_\left(2713+1 \_2714-1\right) \mathrm{del}$ & ex24-26 & p. $\left(\mathrm{R} 830 \mathrm{Sfs}{ }^{*} 14\right)$ & $\mathrm{U}$ & NA & Low \\
\hline $\mathrm{D} / \mathrm{O}$ & 44 & g.78238C > T & c. $1654 C>T$ & ex17 & p. $\left(\mathrm{R} 552^{*}\right)$ & $\mathrm{U}$ & NA & Low \\
\hline $\mathrm{O} / \mathrm{E}$ & 45 & g.76898C>T & c. $1399 \mathrm{C}>\mathrm{T}$ & ex15 & p. $\left(R 467^{*}\right)$ & $\mathrm{U}$ & NA & Low \\
\hline O/D & 57 & g.153352delA & c.1959delA & ex19 & p.(V654Cfs*4) & $\mathrm{U}$ & NA & High \\
\hline $\mathrm{O} / \mathrm{C}$ & 59 & g.65386C $>\mathrm{T}$ & c. $1072 \mathrm{C}>\mathrm{T}$ & ex11 & p. $\left(\mathrm{R} 358^{*}\right)$ & $\mathrm{U}$ & 0.5 & Low \\
\hline O/D & 60 & g.59695C $>\mathrm{T}$ & c.763C>T & ex8 & p. $\left(\mathrm{R} 255^{\star}\right)$ & $\mathrm{U}$ & NA & Low \\
\hline Retinoma/O & 100 & & gainex3_ex23 & $\begin{array}{c}\text { tandem repeat of } \\
\text { ex3_23 at the } \\
\text { transcript level } \\
\end{array}$ & p. (1831Efs $\left.{ }^{\star} 22\right)$ & $U$ & 0.5 & High \\
\hline D/ Retinoma & 117 & g.162093C>T & c. $2308 \mathrm{C}>\mathrm{T}$ & ex22 & p. $\left(Q 770^{*}\right)$ & $\mathrm{B}$ & 2 & High \\
\hline$E / D$ & 24 & g.162069C>T & c. $2284 \mathrm{C}>\mathrm{T}$ & ex22 & p. $\left(Q 762^{*}\right)$ & $\mathrm{B}$ & $\mathrm{NA}$ & High \\
\hline$B / D$ & 24 & g.160785_160791dupTCAAAAT & c.2152_2168dupTCAAAAT & ex21 & p. $\left(1724 Q f s^{*} 29\right)$ & B & 1 & Low \\
\hline O/D & 45 & g. $150037 \mathrm{C}>\mathrm{T}$ & c. $1735 \mathrm{C}>\mathrm{T}$ & ex18 & p. $\left(\mathrm{R} 579^{*}\right)$ & $U$ & NA & Medium \\
\hline O/D & 29 & g. $162237 \mathrm{C}>\mathrm{T}$ & c. $2359 \mathrm{C}>\mathrm{T}$ & ex23 & p. $\left(R 787^{*}\right)$ & $U$ & NA & Low \\
\hline
\end{tabular}

\section{0}

531

532

533

534

535

536

537

538

539

540

541

542

543

544
Table 2c. Genetic mutation data for mosaic carriers of the $R B 1$ mutation. Genomic (g.) nucleotide numbering is according to GenBank sequence accession number L11910.1. In CDNA (c.) nucleotide numbering c.1 is the A of the ATG translation initiation codon based on the Locus Reference Genomic Sequence LRG_517t1 (RB1). Mutation nomenclature is according to Human Genome Variation Society guidelines (www.hgvs.org). ex - exon, int- intron, pro- promoter, sd- splice donor, sa- splice acceptor, g- germline, m- mosaic, U- unilateral, B- bilateral. NA- not applicable 
545

546

547

548 